\title{
ON THE STRUCTURE OF CERTAIN FACTORIZABLE GROUPS. II
}

\section{DANIEL GORENSTEIN AND I. N. HERSTEIN}

1. In [3], we have shown that in a finite $A B$-group $G$ in which $A$ and $B$ are cyclic and $A$ is its own normalizer, the commutator subgroup $T$ of $G$ is cyclic and $G=A T$ with $A \cap T=1$. This result can be used to determine the structure of arbitrary $A B$-groups in which $A$ and $B$ are cyclic.

If $A$ is a subgroup of a group $G$, define the subgroup $N^{i}(A)$ of $G$ inductively by the formula $N^{i}(A)=N_{G}\left(N^{i-1}(A)\right)$, and denote by $N^{*}(A)$ the upper bound of the subgroups $N^{i}(A)$. Using this notation, we shall prove the following theorem concerning $A B$-groups:

Theorem A. Let $G$ be a finite group of the form $A B$, where $A$ and $B$ are cyclic subgroups of $G$. Then $G$ contains a unique cyclic normal subgroup $T$ such that $G=N^{*}(A) T$ and $N^{*}(A) \cap T=1$. Moreover, if $N^{*}(A)=A B^{*}$ with $B^{*} \subset B$, then $B^{*}$ and $T$ commute elementwise.

2. We begin with several lemmas:

Lemma 1. Let $G=A B$, with $A$ and $B$ cyclic, and assume that some subgroup $B^{\prime}$ of $B$ is normal in $G$. Let $\bar{G}=G / B^{\prime}=\bar{A} \bar{B}$, where $\bar{A}, \bar{B}$ are the images of $A, B$ in $\bar{G}$. Then $N_{G}^{*}(A) B^{\prime}$ is the complete inverse image of $N_{\bar{G}}^{*}(\bar{A})$ in $G$.

Proof. Let $B_{0} \subset B^{\prime}$ with $o\left(B_{0}\right)=p$. Since $B^{\prime}$ is cyclic, $B_{0}$ is normal in $G$. If $B_{0}<B^{\prime}$, set $\widetilde{G}=G / B_{0}=\widetilde{A} \widetilde{B}$, and let $\widetilde{B}^{\prime}$ be the image of $B^{\prime}$ in $\widetilde{G}$. Since $\bar{G} \cong \widetilde{G} / \widetilde{B}^{\prime}$, it follows by induction on the order of $G$ that the inverse image of $N_{\tilde{G}}^{*}(\bar{A})$ in $\tilde{G}$ is $N_{\tilde{G}}^{*}(\tilde{A}) \tilde{B}^{\prime}$. Hence to prove the lemma, it suffices to show that $N(\tilde{G} \tilde{A})^{-1}=N_{G}^{*}(A) B_{0}$. Thus without loss of generality we may assume $o\left(B^{\prime}\right)=p$.

Let $A=(a), B=(b)$ and $B^{\prime}=\left(b^{r}\right)$. It is clearly sufficient to prove by induction on $i$ that if $b^{u} \in N^{i}(\bar{A})^{-1}$, then $b^{u} \in N^{i}(A) B^{\prime}$ for some $j$. Now for some integer $\lambda$ with $0<\lambda<p$, we have

$$
a b^{r} a^{-1}=b^{r \lambda} \text {. }
$$

We treat the cases $\lambda=1$ and $\lambda>1$ separately. If $\lambda=1, B^{\prime} \subset N(A)$. Now if $b^{u} \in N^{i}(\bar{A})^{-1}, b^{u} \in N^{i}(\bar{A})$ and hence $\bar{b}^{u} \bar{a} \bar{b}^{-u} \in N^{i-1}(\bar{A})$. By induction $b^{u} a b^{-u} \in N^{j}(A) B^{\prime}$. If $j=0, b^{u} a b^{-u} \in N^{1}(A)$ and consequently $b^{u} \in N^{2}(A)=N^{2}(A) B^{\prime}$. If $j>0, N^{j}(A) B^{\prime}=N^{j}(A)$, and so $b^{u} \in N^{j+1}(A)$ $=N^{i+1}(A) B^{\prime}$.

Received by the editors May 4, 1959. 
If $\lambda>1$, it follows as above that $b^{u} a b^{-u} \in N^{i}(A) B^{\prime}$. If $N^{j}(A)=A B_{j}$ and $B_{j}=\left(b^{s}\right)$, we have

$$
b^{u} a b^{-u}=a^{\alpha} b^{8 \beta} b^{r \gamma} \text { for suitable integers } \alpha, \beta, \gamma .
$$

Since $(\lambda-1, p)=1$, we can find an integer $\delta$ such that $\gamma+\delta \lambda$ $\equiv \delta(\bmod p)$. We then have

$$
a^{-1} b^{u+r \delta} a=\left(a^{-1} b^{u} a\right)\left(a^{-1} b^{r \delta} a\right)=\left(a^{\alpha-1} b^{s \beta+r \gamma+u}\right) b^{r \delta \lambda}=a^{\alpha-1} b^{s \beta} b^{u+r \delta} \text {, }
$$

whence $b^{u+r \delta} \in N^{j}(A)$. Thus $b^{u} \in N^{j}(A) B^{\prime}$, and the lemma is proved.

Lemma 2. Let $G=A B$, with $A$ and $B$ cyclic. Then $G$ contains a cyclic subgroup $T$, invariant under $A$, such that $G=N^{*}(A) T$ and $N^{*}(A) \cap T$ $=1$.

Proof. Either a subgroup of $A$ or a subgroup of $B$ is normal in $G$ (Douglas [1]). Let $A_{1}$ be the maximal subgroup of $A$ normal in $G$, and assume first that $A_{1} \neq 1$. If $\bar{G}=G / A_{1}=\bar{A} \bar{B}$, we may assume by induction that $\bar{G}=N^{*}(\bar{A}) \bar{T}$, where $N^{*}(\bar{A}) \cap \bar{T}=1, \bar{T}$ is cyclic and invariant under $\bar{A}$. Clearly $N^{*}(A)=N^{*}(\bar{A})^{-1}$. If $T_{0}=\bar{T}^{-1}, G=N^{*}(A) T_{0}$ where $N^{*}(A) \cap T_{0}=A_{1}$ and $T_{0}$ is $A$-invariant.

If we let $G_{0}=A T_{0}=A B_{0}$ with $B_{0} \subset B$, it follows from our conditions that $N_{G_{0}}(A)=A$. The proof of Theorem A of [3] now implies that if $T=\left[G_{0}, G_{0}\right]$, then $T \subset T_{0}$, with $T$ cyclic, $G_{0}=A T$ and $A \cap T=1$. It follows at once that $G=N^{*}(A) T$ with $N^{*}(A) \cap T=1, T$ cyclic and invariant under $A$.

If $A_{1}=1$, we consider a minimal subgroup $B^{\prime}$ of $B$ which is normal in $G$; and this time we set $\bar{G}=G / B^{\prime}=\bar{A} \bar{B}$. By induction $\bar{G}=N^{*}(\bar{A}) \bar{T}$, where $\bar{T}$ is cyclic, $\bar{A}$-invariant, and $N^{*}(\bar{A}) \cap \bar{T}=1$. If $T_{0}=\bar{T}^{-1}$, it follows from Lemma 1 that $G=N^{*}(\bar{A})^{-1} T_{0}=N^{*}(A) B^{\prime} T_{0}=N^{*}(A) T_{0}$, where $N^{*}(A) \cap T_{0}=B^{\prime}$. Using the notation of Lemma 1, we consider the cases $\lambda=1$ and $\lambda>1$ separately.

If $\lambda=1$, set $G_{0}=A T_{0}$ and $\bar{G}_{0}=\bar{A} \bar{T}$. By Theorem $\mathrm{A}$ of [3], $\bar{T}$ $=\left[\bar{G}_{0}, \bar{G}_{0}\right]$. Hence we can find a commutator $t$ in $T_{0}$, which maps on a generator $\bar{t}$ of $\bar{T}$. Let $o(\bar{T})=m$ and suppose, if possible, that $t$ has order $m p$. Since $o\left(T_{0}\right)=m p$, it follows that $T_{0}=(t)$, and consequently $T_{0}=\left[G_{0}, G_{0}\right]$. If $a t a^{-1}=t^{\sigma},\left[G_{0}, G_{0}\right]=\left(t^{\sigma-1}\right)$, and hence $(\sigma-1, m p)=1$. But $t^{m} \in B^{\prime}$ and, since $\lambda=1, B^{\prime}$ is in the center of $G$. Thus $t^{m}=a t^{m} a^{-1}$ $=t^{m \sigma}$, whence $p \mid(\sigma-1)$, a contradiction.

If $(t)=\left[G_{0}, G_{0}\right]$, we set $T=(t)$. Since $o(T)=m, T \cap B^{\prime}=1$. Furthermore $T$ is normal in $G_{0}$. We conclude at once that $G=N^{*}(A) T$, $N^{*}(A) \cap T=1, T$ cyclic and invariant under $A$.

On the other hand, if $(t)<\left[G_{0}, G_{0}\right]$, we must have $T_{0}=\left[G_{0}, G_{0}\right]$. Since $G_{0}$ is an $A B$-group, its commutator subgroup $T_{0}$ is abelian (Ito 
[4]). Now $o\left(T_{0}\right)=m p$ and we have just shown that $T_{0}$ contains no commutator of order $m p$. Therefore $p \mid m$.

Since $T_{0}$ is normal in $G_{0}$ and is generated by $t$ and $b^{r}$, we have

$$
a t a^{-1}=t^{\sigma} b^{r \beta} \quad \text { for suitable } \sigma, \beta .
$$

It follows that $\left(\bar{t}^{\sigma-1}\right)=\left[\bar{G}_{0}, \bar{G}_{0}\right]$ and hence that $(\sigma-1, m)=1$. Since $p \mid m$, there exists an integer $\alpha$ such that $\beta+\alpha \equiv \alpha \sigma(\bmod p)$. Consequently $a\left(t b^{r \alpha}\right) a^{-1}=t^{\sigma} b^{r \beta} b^{r \alpha}=t^{\sigma} b^{r \alpha \sigma}=\left(t b^{r \alpha}\right)^{\sigma}$. It follows that the subgroup $T=\left(t b^{r \alpha}\right)$ is invariant under $A$. Since $o(T)=m, T \cap B^{\prime}=1$, and we conclude at once that $G=N^{*}(A) T, N^{*}(A) \cap T=1, T$ cyclic and invariant under $A$.

If $\lambda>1$, we set $T=\left[G_{0}, G_{0}\right]$. Since $T \subset T_{0}, N^{*}(A) \cap T \subset B^{\prime}$. Suppose, if possible, that $B^{\prime} \subset N^{*}(A)$, and let $d$ be the least integer such that $B^{\prime} \subset N^{d}(A)$. By definition of $N^{d}(A), b^{r} a^{-1} b^{-r} \in N^{d-1}(A)$, and hence $a b^{r} a^{-1} b^{-r}=b^{r(\lambda-1)} \in N^{d-1}(A)$. Since $(\lambda-1, p)=1$, it follows that $b^{r} \in N^{d-1}(A)$, a contradiction. Thus $B^{\prime} \cap N^{*}(A)=1$, and consequently $N^{*}(A) \cap T=1$. On the other hand, by Theorem A of [3], $T$ is cyclic and $G_{0}=A T$. We conclude that in all cases $G$ contains a cyclic subgroup $T$, invariant under $A$, such that $G=N^{*}(A) T$ and $N^{*}(A) \cap T$ $=1$.

Lemma 3. Let $G=A B=N^{*}(A) T$ with $N^{*}(A) \cap T=1$, where $T$ is cyclic and $A$-invariant and assume that $A \cap B=1$. If $N^{*}(A)=A B^{*}$ and $A T=A B_{0}$ with $B^{*}, B_{0} \subset B$, then $\left(o\left(B^{*}\right), o\left(B_{0}\right)\right)=1, B=B^{*} \times B_{0}$, and $o(T)=o\left(B_{0}\right)$.

Proof. $G=N^{*}(A) T=\left(A B^{*}\right) T=\left(A B^{*}\right)(A T)=\left(A B^{*}\right)\left(A B_{0}\right)$ $=A\left(B^{*} B_{0}\right)$. Since $A \cap B=1$, it follows that $B=B^{*} B_{0}$. On the other hand, $\quad N^{*}(A) \cap T=1, \quad N^{*}(A) \cap A T=A, \quad$ and hence $N^{*}(A) \cap B_{0}$ $\subset A \cap B_{0}=1$. Thus $B^{*} \cap B_{0}=1$, whence $B=B^{*} \times B_{0}$. Since $B^{*}$ and $B_{0}$ are subgroups of the cyclic group $B$, it also follows that $\left(o\left(B^{*}\right), o\left(B_{0}\right)\right)=1$.

Finally let $T=(t)$, where $t=a^{s} b^{r}$ and let $o(T)=m$. Since $A T=A B_{0}$, it follows as in the proof of Theorem 10 of [2] that $T$ consists of the elements $a^{s i} b^{r j}$, and since $A \cap B=1$, these elements must be distinct for $j=1,2, \cdots, m$ and $a^{s m} b^{r m}=1$. Hence $b^{r m}=1$ and so $o\left(B_{0}\right) \mid m$. On the other hand, if $o\left(B_{0}\right)=n<m, a^{n} b^{r n}=a^{s n} \in A \cap T=1$, whence $a^{s n} b^{r n}=a^{s m} b^{r m}$, a contradiction. Thus $o\left(B_{0}\right)=o(T)$, as asserted.

Lemma 4. $T$ is uniquely determined by the conditions $G=A B$ $=N^{*}(A) T$ with $N^{*}(A) \cap T=1, T$ cyclic and $A$-invariant.

Proof. Suppose $T, T^{\prime}$ are two subgroups of $G$ satisfying the conditions of the lemma. Let $G_{0}=A T$ and $G_{0}^{\prime}=A T^{\prime}$. Since $A \cap T=1$, 
$N_{G_{0}}(A)=A$, whence by Theorem A of [3], $T=\left[G_{0}, G_{0}\right]$, and similarly $T^{\prime}=\left[G_{0}^{\prime}, G_{0}^{\prime}\right]$. Hence to prove the lemma, it clearly suffices to show that $G_{0}=G_{0}^{\prime}$.

If $A \cap B \neq 1$, the equality of $G_{0}$ and $G_{0}^{\prime}$ follows readily by induction by considering $\bar{G}=G / A \cap B$; hence without loss of generality we may assume that $A \cap B=1$. If $G_{0}=A B_{0}$ and $G_{0}^{\prime}=A B_{0}^{\prime}$, it follows from Lemma 3 that $B=B^{*} \times B_{0}$ and $B=B^{*} \times B_{0}^{\prime}$. Hence $o\left(B_{0}\right)=o\left(B_{0}^{\prime}\right)$. But $B$, being cyclic, has a unique subgroup of any given order. Thus $B_{0}=B_{0}^{\prime}$ and $G_{0}=G_{0}^{\prime}$.

3. Proof of Theorem A. In view of Lemmas 2 and 4 it suffices to prove that $T$ commutes elementwise with $B^{*}$, for this will clearly imply that $T$ is normal in $G$. In this section we treat the case $A \cap B$ $=1$.

Let $d$ be the least integer such that $N^{d+1}(A)=N^{d}(A)$, so that $N^{*}(A)=N^{d}(A)$. Let $N^{i}(A)=A B_{i}$ with $B_{i}=\left(b^{r_{i}}\right) \subset B, i=1,2, \cdots, d$. Then $B_{1}<B_{2}<\cdots<B_{d}$ and $B_{d}=B^{*}$. We may assume $r_{i} \mid r_{i-1}, i=2$, $3, \cdots, d . N^{i-1}(A)$ is normal in $N^{i}(A)$ since $N^{i}(A)=N_{G}\left(N^{i-1}(A)\right)$. Furthermore let $G_{0}=A T=A B_{0}$ with $B_{0}=\left(b^{r}\right) \subset B$ and $o\left(B_{0}\right)=m$. Then $T=(t)$, where $t=a^{8} b^{r}$ for some integer $s$.

If $s=0, T=B_{0}$, and it is obvious that $T$ and $B^{*}$ commute elementwise. Hence we may suppose $s \neq 0$ and without loss of generality that $s \mid h$, where $h=o(A)$. First of all, if $h<s m, a^{h} b^{r h / s}=b^{r h / s} \in T$, and generates a subgroup $T_{0}$, which is clearly invariant under $B$ and hence is normal in $G$. It follows at once by considering $G / T_{0}$ and using induction on the order of $G$, that

$$
b^{r d} t b^{-r_{d}}=t b^{r(h / s) \beta} \quad \text { for some integer } \beta .
$$

If $n$ denotes the order of $B^{*}$, we conclude at once from (4) that $t=b^{r d n} t b^{-r d n}=t b^{r(h / s) \beta n}$, whence

$$
r(h / s) \beta n \equiv 0(\bmod m) .
$$

Since $(n, m)=1$ by Lemma 3 ,

$$
r(h / s) \beta \equiv 0(\bmod m) \text { and } b^{r^{d}} l b^{-r_{d}}=t \text {, as desired. }
$$

We may therefore assume that $h=s m$. For $i=1,2, \cdots, d$ we have (6) $b^{r_{i}} a b^{-r_{i}}=a^{u_{i-1}} b^{r_{i-1} v_{i-1}}$ for suitable integers $u_{i-1}, v_{i-1}$, where $r_{0}=0$.

Let $G_{i}^{\prime}$ be the commutator subgroup of $G_{i}=N^{i}(A) T$. We know that $T=(t)$ is the commutator subgroup of $G_{0}=A T$. Since $N^{i-1}(A)$ is normal in $N^{i}(A)$, and $G_{i}=N^{i}(A) B_{0}, G_{i-1}$ is normal in $G_{i}$. It follows readily by induction that $G_{i}^{\prime}$ is generated by the elements $a^{u_{0}-1}$, 
$a^{u_{1}-1} b^{r_{1} v_{1}}, \cdots, a^{u_{i-1}-1} b^{r_{i-1} v_{i-1}}, t$. Furthermore $G_{i}^{\prime}$ is abelian since $G_{i}$ is an $A B$-group for each $i$.

To prove that $B^{*}$ and $T$ commute elementwise, we have only to show that $b^{r i} t b^{-r_{i}}=t$ on the assumption that $b^{r_{i-1}} t b^{-r_{i-1}}=t$. Now from the form of $G_{i}^{\prime}$, we have

$$
b^{r_{i}} t b^{-r_{i}}=x t^{\gamma} \text { where } x \in N^{i-1}(A) \text { and } x t=t x .
$$

Since by assumption $A \cap B=1$, Lemma 3 implies $o(T)=o\left(B_{0}\right)$, whence $t^{m}=1$. It follows now from (7) that $x^{m}=1$. Suppose for some $j>1, x \in N^{i}(A), x \notin N^{j-1}(A)$. Let $\beta$ be the least integer such that $x^{\beta} \in N^{j-1}(A)$. Since $N^{j-1}(A)$ is normal in $N^{j}(A) \beta \mid\left[N^{j}(A): N^{j-1}(A)\right]$ and hence $\beta \mid o\left(B^{*}\right)=n$. But clearly $\beta \mid m$ since $x^{m}=1$. Since $(n, m)=1$, $\beta=1$ and so $x \in N^{j-1}(A)$, a contradiction. Thus $x \in A$ and (7) takes the form

$$
b^{r_{i}} t b^{-r_{i}}=a^{\rho} t^{\gamma}, \quad a^{\rho} t=t a^{\rho} .
$$

Now $t=a^{s} b^{r}$ and $t^{\gamma}=a^{s \sigma} b^{r \sigma}$ for some integer $\sigma$, whence $b^{r_{i}} a^{s} b^{-r_{i}}$ $=a^{\rho+8 \sigma} b^{r(\sigma-1)}$. But this implies $b^{r(\sigma-1)} \in N^{i-1}(A) \cap B_{0}=1$, so that $\sigma \equiv 1(\bmod m)$. Since $a^{s m}=1$, we may assume $\sigma=\gamma=1$, and hence that

$$
b^{r_{i}} a^{s} b^{-r_{i}}=a^{\rho+s}, \quad b^{r_{i}} t b^{-r_{i}}=a^{\rho} t .
$$

In particular, (9) implies that $s \mid \rho$.

Since $T$ is normal in $A T$, we have finally

$$
a t a^{-1}=t^{\lambda} \quad \text { for some integer } \lambda \text {. }
$$

In view of (6)

$$
\left(b^{r_{i}} a\right) t\left(b^{r_{i}} a\right)^{-1}=\left(a^{u_{i-1}} b^{r_{i}+r_{i-1} v_{i-1}}\right) t\left(a^{u_{i}-1} b^{r_{i}+r_{i-1} v_{i-1}}\right)^{-1} .
$$

Using (9) and (10) and our assumption that $b^{r^{i-1}}$ commutes with $t$, we conclude readily from (11) that

$$
a^{\rho \lambda} t^{\lambda}=a^{\rho} t^{\lambda^{u_{i}-1}} \text {. }
$$

Since $A \cap T=1, \rho(\lambda-1) \equiv 0(\bmod h)$. Since $h=m s$ and $s \mid \rho$, we obtain

$$
\frac{\rho}{s}(\lambda-1) \equiv 0(\bmod m)
$$

But $T$ is the commutator subgroup of $A T$, which implies $(\lambda-1, m)$ $=1$; and it follows from $(13)$ that $\rho \equiv 0(\bmod h)$. Hence $b^{r i} t b^{-r_{i}}=t$, as desired. We conclude that $B^{*}$ and $T$ commute elementwise.

4. Finally we treat the case $A \cap B \neq 1$. Let $\bar{G}=G / A \cap B=\bar{A} \bar{B}$ 
$=N^{*}(\bar{A}) \bar{T}$, where $\bar{A}, \bar{B}, \bar{T}$ are the images of $A, B, T$, in $\bar{G}$. Clearly $\bar{T}$ is cyclic, invariant under $\bar{A}$, and $N^{*}(\bar{A}) \cap \bar{T}=1$. If $N^{*}(\bar{A})=\bar{A} \bar{B}^{*}$ with $\bar{B}^{*} \subset \bar{B}$, let $n=o\left(\bar{B}^{*}\right)$; and let $m=o(\bar{T})$. Since $\bar{A} \cap \bar{B}=1$, it follows from the preceding section that $(n, m)=1$ and that $\bar{B}$ commutes elementwise with $\bar{T}$. Furthermore $N^{*}(\bar{A})^{-1}=N^{*}(A)(A \cap B)=N^{*}(A)$, and hence $B^{*}$ is the inverse image of $\bar{B}^{*}$ in $G$. If $B^{*}=\left(b^{r} d\right)$, it follows that

$$
b^{r_{d}} t b^{-r_{d}}=x t, \quad x \in A \cap B, \text { and } b^{r_{d} n} \in A \cap B .
$$

Since $A \cap B$ is in the center of $G, x^{m}=1$. On the other hand (14) yields $t=b^{r_{d} n} t b^{-r_{d n}}=x^{n} t$, whence $x^{n}=1$. Since $(n, m)=1$, we conclude that $x=1$; and the theorem is proved.

\section{BIBLIOGRAPHY}

1. J. Douglas, On finite groups with 2 independent generators. I, Proc. Nat. Acad. Sci. U.S.A. vol. 37 (1951) pp. 604-610.

2. D. Gorenstein, Finite groups which admits an automorphism with few orbits, Canad. J. Math. vol. 12 (1960) pp. 73-100.

3. D. Gorenstein and I. N. Herstein, On the structure of certain factorizable groups. I, Proc. Amer. Math. Soc. vol. 10 (1959) pp. 940-945.

4. N. Ito, Products of Abelian groups, Math. Z. vol. 62 (1955) pp. 400-401.

Clark University and

Cornell University 